\title{
Des établissements « branchés 》 - I
}

Une école primaire au Nouveau-Brunswick

\section{Francine Julien}

\section{(2) OpenEdition}

\section{Journals}

Édition électronique

URL : http://journals.openedition.org/ries/2911

DOI : 10.4000/ries.2911

ISSN : 2261-4265

Éditeur

Centre international d'études pédagogiques

Édition imprimée

Date de publication : 1 juin 1998

Pagination : 67-71

ISSN : $1254-4590$

Référence électronique

Francine Julien, « Des établissements «branchés » - I », Revue internationale d'éducation de Sèvres [En ligne], 18 | juin 1998, mis en ligne le 19 avril 2013, consulté le 02 mai 2019. URL : http:// journals.openedition.org/ries/2911; DOI : 10.4000/ries.2911

Ce document a été généré automatiquement le 2 mai 2019.

(c) Tous droits réservés 


\title{
Des établissements « branchés » - I
}

\author{
Une école primaire au Nouveau-Brunswick
}

\author{
Francine Julien
}

1 L'intégration des nouvelles technologies de l'information et des communications constitue une importante préoccupation dans le monde de l'éducation. La connaissance et l'utilisation des nouvelles technologies sont, sans contredit, jugées essentielles pour survivre dans un monde de plus en plus informatisé; pourtant plusieurs questions se posent aux intervenants du monde scolaire. La définition de l'apprentissage à l'aide des nouvelles technologies, la difficulté du choix des indicateurs qui permettent de juger de la qualité et de l'efficacité de l'apprentissage, le problème de l'intégration d'un environnement technologique dans les programmes existants, la difficulté de redéfinir les grandes stratégies éducatives en fonction des nouvelles réalités sont autant de questionnements qui peuvent faire obstacle.

2 L'utilisation des technologies ne doit pas être indépendante des finalités de l'école et l'action doit être orientée dans la perspective de développer chez les élèves les « habiletés ${ }^{1}$ » dont ils auront besoin. Comment ces habiletés sont-elles développées et soutenues par la technologie et de quelle manière le système scolaire peut-il appuyer un développement approprié et assurer à chaque enfant la possibilité d'acquérir ces habiletés au moyen des nouvelles technologies?

3 Cet article présente un projet pilote d'intégration des nouvelles technologies dans une école du Nouveau-Brunswick, province située à l'est du Canada. La conception d'une nouvelle vision de l'apprentissage est au cœur de ce projet qui a pris naissance en septembre 1997 au Centre d'apprentissage du Haut-Madawaska, une école primaire de 350 élèves. Située dans le village de Clair ${ }^{2}$, petite communauté rurale du Nouveau-Brunswick, l'école regroupe les enfants des régions environnantes de la maternelle à la huitième année. La construction de cette nouvelle école a été l'occasion d'intégrer les nouvelles technologies de l'information et des communications dans le quotidien des élèves et du personnel enseignant.

4 Ce projet d'école «branchée» s'inscrit dans la stratégie pour l'intégration de la technologie dans le système d'éducation que s'est donnée la province du NouveauBrunswick. Mise en place en 1996, cette stratégie a pour but de permettre à chaque élève 
et à chaque éducateur d'avoir accès à des technologies de l'information et des communications actuelles et performantes et de leur faire acquérir les connaissances, les habiletés et les attitudes requises pour les utiliser. Son but est de donner aux éducateurs la formation requise pour intégrer ces technologies dans une diversité de pratiques pédagogiques et dans l'ensemble du curriculum et d'intégrer les technologies de l'information et des communications dans le développement, le contenu et l'enseignement de tous les programmes d'études.

5 Le Centre d'apprentissage du Haut-Madawaska offre à ses enseignants et à ses élèves des ressources matérielles et humaines qui favorisent l'utilisation des nouvelles technologies en travaillant en collaboration, entre autres, avec le ministère de l'Éducation, la compagnie de téléphone NBTEL et les ressources de la communauté. Ces efforts concertés ont permis d'établir des conditions d'apprentissage adaptées aux nouvelles réalités malgré les coûts élevés que comporte normalement un tel projet.

6 Dans cette école, le cheminement de l'élève s'articule autour d'un environnement technique qui l'amène à s'engager personnellement dans une démarche d'appropriation des moyens mis à sa disposition.

7 Les ordinateurs sont présents dans la salle de classe et un local équipé de plusieurs postes d'ordinateurs peut être réservé par les enseignants pour une classe ou un groupe d'élèves. Cette salle sera bientôt munie d'un équipement multimédia permettant aux élèves de créer et d'apprendre dans un environnement médiatisé.

8 La bibliothèque, que l'on nomme ici la Cybertec, est informatisée et la recherche de documents peut se faire directement à partir de l'ordinateur de la salle de classe. Les élèves ont un accès contrôlé à Internet pour effectuer des recherches personnelles et sont branchés sur un système de courrier électronique interne avec lequel ils peuvent adresser du courrier aux autres élèves de l'école, aux enseignants et même au directeur. Tout l'équipement est compatible afin d'éviter les interférences et les problèmes techniques et l'école dispose d'un accès à Internet sur bande élargie.

9 Toutefois, selon le directeur, l'élaboration d'une démarche d'apprentissage dans un tel environnement s'avère un processus complexe. La progression doit être conçue de diverses façons car dans un contexte éducatif renouvelé la pratique doit relever d'une méthodologie bien arrêtée. Il faut dépasser le niveau technique pour donner une perspective particulière à la pratique pédagogique.

10 Actuellement, l'un des plus grands défis est la formation des enseignants, parce que plusieurs d'entre eux craignent d'utiliser l'ordinateur ou ne sont pas encore convaincus que les nouvelles technologies peuvent les aider dans leur enseignement. Bien souvent, l'enthousiasme de faire partie d'une école "branchée » est largement tempéré par le manque de ressources offertes aux enseignants et aux administrateurs scolaires pour les guider dans leurs choix pédagogiques. Les enseignants ne deviennent pas de «meilleurs enseignants » simplement parce qu'ils utilisent les nouvelles technologies.

11 La réussite du projet au Centre d'apprentissage du Haut-Madwaska dépend de l'attention spéciale qui est accordée à l'initiation des enseignants et à l'organisation scolaire et, plus spécifiquement encore, à la gestion pédagogique de la classe. Les diverses applications des nouvelles technologies s'appuient sur la pédagogie actuelle, fondée sur les centres d'apprentissage et les centres d'intérêts, qui se prête bien à leur intégration.

12 Des conditions générales favorables sont obtenues par des stratégies d'enseignement collectives appuyées sur du matériel pédagogique approprié et, surtout, le soutien de 
conseillers spécialisés auprès des enseignants et des élèves. En plus de l'agent pédagogique, un " mentor informatique ${ }^{3}$ " visite l'école du Haut-Madawaska quatre jours par semaine et travaille en collaboration avec les enseignants à préparer des scénarios d'apprentissage intégrant l'utilisation des nouvelles technologies. Chaque enseignant reçoit un document préparatoire qui contient des idées, des démarches pédagogiques et des aides à la recherche sur Internet.

Le rôle principal du mentor est d'aider les enseignants hésitants ou qui manquent de confiance à se servir adéquatement des outils technologiques pour atteindre les objectifs pédagogiques. Il établit un climat favorable et aide à développer une attitude positive face aux nouvelles technologies chez l'ensemble des intervenants.

Plus précisément, le mentor prépare et rédige des plans avec des objectifs spécifiques et mesurables, il définit des moyens de réalisation et rend compte de la situation d'utilisation des ordinateurs par les élèves et les enseignants tout au long des projets. Il est présent en salle de classe et amène l'enseignant à déterminer les activités et les moyens qui permettront l'intégration des technologies à l'intérieur des programmes d'études existants. Il encourage l'enseignant à développer des stratégies d'enseignement, le travail sur projet et une gestion de classe qui permet aux élèves d'accomplir un travail signifiant et valorisant avec une utilisation maximale du matériel technologique.

Le rôle du mentor est également d'amener l'enseignant à exprimer ses propres besoins de formation afin d'intégrer les nouvelles technologies dans sa pratique pédagogique. Ainsi les enseignants en viennent à développer des activités et des projets à partir des objectifs du programme d'études, à entreprendre des recherches, à mettre sur pied des projets d'échanges entre élèves à l'aide du courrier électronique et à développer eux-mêmes du matériel pédagogique à l'aide des outils technologiques.

Les enseignants sont appuyés dans leur démarche et les scénarios d'apprentissage sont bâtis de manière à atteindre des résultats concrets.

$17 \mathrm{Au}$ Centre d'apprentissage du Haut-Madawaska, tous les élèves, de la maternelle à la huitième année, sont initiés à un logiciel de traitement de texte en réalisant des productions. Les plus jeunes ont appris le clavier et la manipulation de la souris en créant des dessins, des signets et des cartes de souhaits. Les plus âgés ont participé à des projets d'écriture plus élaborés, tel un journal de classe contenant des jeux et des devinettes, un livret d'histoires ou un magazine thématique produit en équipe.

18 L'apprentissage des logiciels et des outils de recherche permet aux élèves une maîtrise des moyens techniques et leur assure une certaine autonomie. Puis les enseignants leur fournissent des marches à suivre, des pistes de recherche, pour les aider à intégrer la technologie dans leurs projets, pour leur apprendre à réfléchir à leur message ou leur permettre de découvrir des déclencheurs dans les tâches plus complexes.

Ce renouveau pédagogique développe la coopération entre les élèves et augmente l'estime de soi. Par exemple, les élèves du programme de français accéléré ont préparé des livrets d'histoires illustrés à partir d'un logiciel d'aide à l'écriture pour aider des élèves d'une autre classe présentant des difficultés en lecture. Dans une classe de mathématique, un groupe d'élèves a effectué un sondage auprès des collègues, exécuté des calculs statistiques et produit des tableaux graphiques à l'aide d'un chiffreur électronique. Un logiciel de présentation graphique a ensuite permis au groupe de présenter les données et les conclusions du projet sur écran. 
20 L'utilisation des technologies motive énormément les élèves qui aiment planifier les projets, travailler en équipe et présenter des produits à leurs enseignants et à leurs parents. Toutefois, il faut assurer à chaque élève le temps et les modalités d'apprentissage nécessaires à la maîtrise des objectifs du cours et du programme d'études. La difficulté est de définir des points de repère car l'on passe un certain temps au début à apprendre l'utilisation des logiciels et à préparer des démarches et des scénarios d'apprentissage. Les enseignants et les administrateurs scolaires du Centre sont en train de bâtir des démarches pédagogiques qui favoriseront les processus d'apprentissage à l'aide des nouvelles technologies et la nécessité d'élaborer une méthodologie exige de prolonger au besoin le temps d'apprentissage de chaque élève.

21 Les modèles permettant de vérifier la réussite ou l'échec de l'apprentissage à l'aide des nouvelles technologies sont en voie de construction. De même qu'il n'existe pas de modèle unique qui englobe toutes les dimensions de l'acte d'apprendre, il faudra développer des démarches pédagogiques diversifiées. À cet égard, le Centre d'apprentissage du Haut-Madawaska s'emploie à développer un protocole d'entente avec l'université de Moncton au Nouveau-Brunswick à titre de centre de recherche-action permettant aux futurs enseignants de venir observer les élèves et d'expérimenter de nouvelles approches et de nouveaux produits éducatifs.

Le Centre d'apprentissage du Haut-Madawaska offre un bon exemple d'intégration des nouvelles technologies, car on cherche d'abord à y développer l'intérêt des enseignants qui n'ont pas toujours été préparés au nouveau mode de gestion pédagogique qu'implique l'utilisation des technologies de l'information et des communications dans l'enseignement. Sans appui, les enseignants risquent de se retrouver devant des difficultés insurmontables, pris entre les exigences des programmes d'études de plus en plus chargés, les besoins des élèves et les demandes des parents et des administrateurs.

23 Avec le temps et l'aide qui leur est accordée, les enseignants pourront guider les élèves à franchir avec succès le passage du «puis-je utiliser l'ordinateur et les médias? » au « comment la technologie et les médias peuvent-ils m'aider à apprendre?».

\section{NOTES}

1. Le terme « habileté » renvoie à la notion anglo-saxonne d'« ability», plus souvent exprimée en France sous le terme de « capacité » $(N d l R)$.

2. Centre d'apprentissage du Haut-Madawaska, Clair, Nouveau-Brunswick.

3. Rôle et responsabilités des mentors informatiques, District scolaire 3, Nouveau-Brunswick, 1997. 


\section{RÉSUMÉS}

Le projet pilote d'intégration des nouvelles technologies, mené dans une petite communauté rurale du Nouveau-Brunswick, au Canada, démontre l'importance de l'initiation des enseignants à ces nouveaux outils pour aboutir à une gestion pédagogique efficace de la classe et permettre la mise en place de stratégies d'enseignement renouvelées.

\section{INDEX}

Index géographique : Canada

Mots-clés : école primaire, nouvelles technologies, pédagogie

\section{AUTEUR}

\section{FRANCINE JULIEN}

Coordonnatrice de la formation et de l'évaluation linguistique, Edmundston, Nouveau-Brunswick, Canada 